\title{
Determination of the optimum sampling frequency of noisy images by spatial statistics
}

\author{
Luis Miguel Sanchez-Brea and Eusebio Bernabeu
}

\begin{abstract}
In optical metrology the final experimental result is normally an image acquired with a CCD camera. Owing to the sampling at the image, an interpolation is usually required. For determining the error in the measured parameters with that image, knowledge of the uncertainty at the interpolation is essential. We analyze how kriging, an estimator used in spatial statistics, can generate convolution kernels for filtering noise in regularly sampled images. The convolution kernel obtained with kriging explicitly depends on the spatial correlation and also on metrological conditions, such as the random fluctuations of the measured quantity, and the resolution of the measuring devices. Kriging, in addition, allows us to determine the uncertainty of the interpolation, and we have analyzed it in terms of the sampling frequency and the random fluctuations of the image, comparing it with Nyquist criterion. By use of kriging, it is possible to determine the optimum-required sampling frequency for a noisy image so that the uncertainty at interpolation is below a threshold value. () 2005 Optical Society of America
\end{abstract}

OCIS codes: $\quad 040.1520,100.2650,100.2960,110.4280,120.3940$.

\section{Introduction}

In optical metrology, normally the final experimental result is an image. For example, in interferometry, diffractometry, photoelasticity, and moiré deflectometry, a fringe pattern is obtained, and it is usually acquired with a charge-coupled device (CCD) camera. The finite resolution of these devices, owing to both the practical limitations of their construction and the need to gather sufficient light, produces a sampling in the image. When subpixel resolution is required, an interpolation process must be performed. For that goal, there exist numerous techniques such as linear and polynomial interpolations, convolution kernels, and splines. ${ }^{1}$ The convolution of the experimental data with an interpolation kernel is one of the most effective techniques because of its reliability and

When this research was performed, L. M. Sanchez-Brea (sanchezbrea@fis.ucm.es) was with the Departamento de Optica, Universidad Complutense de Madrid, Escuela Universitaria de Optica, Arcos del Jalon s/n. 28037 Madrid, Spain, and E. Bernabeu was with the Departamento de Optica, Universidad Complutense de Madrid, Facultad de Ciencias Fisicas, Ciudad Universitaria s/n., 28040 Madrid, Spain. L. M. Sanchez-Brea is currently contracted by the Universidad Complutense de Madrid within the Ramón y Cajal program, of the Ministerio de Educación y Ciencia of Spain.

Received 29 July 2004; revised manuscript received 29 November 2004; accepted 29 November 2004.

0003-6935/05/163276-08\$15.00/0

(C) 2005 Optical Society of America short computing time, which is still a research

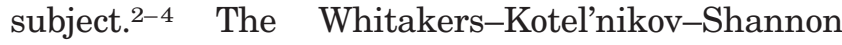
technique 5 (WKS) has been a milestone in signal processing, proposing the sinc function as convolutional kernel. However, WKS requires a regular and infinite sampling, and the signal needs to be band limited. As a consequence of so restrictive conditions, the reconstruction of the sampled signal is exact, and the uncertainty proposed by WKS is 0 when the sampling frequency is at least twice the highest frequency at the image.

When one measures with a CCD camera, noise appears, ${ }^{6}$ and WKS does not provide a good interpolation because the estimation at the sampling location is exactly the measured value, including noise. Many efforts have been made to get other convolutional kernels ${ }^{1,7-10}$ that allow noise filtering, such as Gaussian and cubic B spline. A common property of all these interpolation methods is that they do not estimate the interpolation uncertainty. However, when the image is used for optical metrology, the error in the parameters measured with the image needs to be calculated, and knowledge of this interpolation uncertainty is therefore crucial.

The standard technique for determining the uncertainty of a quantity $Q$ is to obtain a number of measurements under the same conditions. The most accurate prediction of $Q$ is its arithmetic mean $(\bar{Q})$, and the uncertainty at the estimation is given by 


$$
\sigma(\bar{Q})=\left[I^{2}+\frac{s^{2}(Q)}{N}\right]^{1 / 2}
$$

where $N$ is the number of measurements, $s^{2}(Q)$ is the experimental variance, and $I$ is the resolution of the measuring device. ${ }^{11,12}$ This procedure of noise filtering and uncertainty calculation is not normally used in image processing because several images should be acquired. In addition, it cannot be applied in realtime applications, and the acquisition time and computing resources increase considerably.

The convolutional methods use the data around the location $x$ at which interpolation is performed for filtering noise. However, the spatial correlation should be considered for this because, otherwise, data obtained at different locations represent independent processes. Other parameters that should be considered for the interpolator design are the random fluctuations of the measured quantity and the resolution of the measuring devices.

A technique to estimate a quantity with spatial dependence and its uncertainty from the experimental data that explicitly considers the spatial correlation is kriging, ${ }^{13,14}$ which is a family of best linear unbiased estimators in the minimal squared sense. Kriging is widely used in geostatistics and other experimental sciences such as geology, mining, biology, and medicine, for which the available data values are few, are irregularly distributed in space, and present strong random fluctuations. Kriging has also been applied to image processing. ${ }^{15,16}$ However, in its general form, it requires the inversion of matrices with a size equal to the number of data values (that for a bidimensional CCD camera is around $10^{5}-10^{6}$ ). Recently kriging has aroused interest for image processing because researchers have shown that, for regularly sampled data, it can be solved with a convolution and consideration of the finite size of the pixels. ${ }^{17}$ Therefore the computing time is considerably reduced. In addition, kriging has been proven to obtain better results than the adaptive Wiener filter in image processing. ${ }^{18}$

In this paper we show that kriging is a valuable technique in processing images, reducing noise, and determining the interpolation uncertainty. In Section 2 we discuss how kriging can be adapted for its use in image processing. In Section 3 we analyze the capabilities of kriging for the uncertainty determination, and we compare it with the well-known WKS method. We show that the interpolation performed with kriging presents a kind of Nyquist criterion: When the sampling frequency is lower than twice the highest frequency of the noiseless signal, the estimated uncertainty is high; it decreases considerably when the sampling frequency is greater. However, the transition zone between these two cases is continuous, and the width of such a transition zone depends on the noise. Although one can reduce the uncertainty calculated with the kriging technique by increasing the sampling frequency, it is never lower than the resolution of the measuring devices. In Section 4 we apply kriging to experimental two-dimensional (2D) images obtained with the shadow moiré technique, and we present a procedure to determine the optimum sampling frequency so that the interpolation of the image has an uncertainty lower than a threshold value that is presented.

\section{Kriging with Noisy Regularly Sampled Data}

Kriging is a family of linear methods for the estimation of physical quantities with spatial dependence $Z(\mathbf{x})\left(\mathbf{x} \subset \mathfrak{R}^{D}\right)$ - with $D$ as the dimension-and its uncertainty $\sigma(\mathbf{x})$ from $N$ data $Z_{i}$ placed at $\mathbf{x}_{i}, i$ $=1, \cdots, N$, which are optimum in the squared minima sense. The resolution of the measuring devices is $I_{i}, i=1, \cdots, N$. In performing the interpolation, kriging considers the spatial correlation of the quantity by means of a variogram, which is defined as

$$
2 \gamma(h)=E\left[\left(Z_{i}-Z_{j}\right)^{2}\right]=\frac{1}{N(h)} \sum_{i=1}^{N(h)}\left(Z_{i}-Z_{j}\right)^{2},
$$

where $Z_{i}$ and $Z_{j}$ are the experimental data values at $\mathbf{x}_{i}$ and $\mathbf{x}_{j}$, respectively, and $N(h)$ is the number of different pairs $\left(\mathbf{x}_{i}, \mathbf{x}_{j}\right)$ whose distance is $h$. The variogram is closely related to covariance $C(h)$, and when $Z(\mathbf{x})$ is stationary of second order, then $\gamma(h)=C(0)$ - $C(h)$. For simplicity, we have assumed that the quantity with spatial dependence $Z(\mathbf{x})$ is isotropic so that the spatial correlation depends only on the distance between locations. When this assumption is not considered, then the variogram depends on a vector variable $\gamma(\mathbf{h})$. We also assume that $Z(\mathbf{x})$ presents a spatial dependence that can be described as a linear combination of known functions $f_{0}(\mathbf{x}), \ldots, f_{p}(\mathbf{x})$ :

$$
m(\mathbf{x})=E[Z(\mathbf{x})]=\sum_{l=0}^{p} \beta_{l} f_{l}(\mathbf{x}),
$$

where $f_{0}(\mathbf{x})=1$. In an optical image, $m(\mathbf{x})$ may represent a nonuniform illumination, and when it is considered (universal kriging), kriging can decrease the uncertainty in the estimation. If there is not knowledge of such long-range variability (ordinary kriging), then it can be assumed constant $\left[m(\mathbf{x})=\beta_{0}\right]$. In addition to the spatial dependence $m(\mathbf{x}), Z(\mathbf{x})$ presents a random fluctuation $e(\mathbf{x})$ :

$$
Z(\mathbf{x})=m(\mathbf{x})+e(\mathbf{x}),
$$

where $E(e)=0$ and its standard deviation is $E\left(e^{2}\right)$ $=s$. The interpolated value at a given location $\mathbf{x}$ is obtained as a linear combination of the measured values $Z_{i}$ :

$$
Z(\mathbf{x})=\sum_{i=1}^{N} \lambda_{i}(\mathbf{x}) Z_{i}
$$

where $\lambda_{i}(\mathbf{x})$ are the weights to be calculated.

Let $Z_{0}(\mathbf{x})$ be the noiseless version of $Z(\mathbf{x})$. One performs the estimation by minimizing the squared 
mean of the difference:

$$
\sigma^{2}(\mathbf{x})=E\left\{\left[Z(\mathbf{x})-Z_{0}(\mathbf{x})\right]^{2}\right\}
$$

where unbiasedness is imposed through Lagrange multipliers

$$
\sum_{i=0}^{N} \lambda_{i}(\mathbf{x}) f_{l}(\mathbf{x})=1, \quad l=0, \ldots, p .
$$

One performs minimization of Eq. (6) by deriving $\sigma^{2}(\mathbf{x})$ with respect to $\lambda_{i}(\mathbf{x})$ and $\beta_{l}$ and equaling each derivation to zero. Then a linear system of equations is obtained from which we can obtain the value of the $\lambda_{i}(\mathbf{x})$ parameters ${ }^{14}$ :

$$
\lambda^{T}(\mathbf{x})=[\gamma+\mathbf{F H} g]^{T} \boldsymbol{\Gamma}^{-1},
$$

where ${ }^{T}$ means transpose,

$$
\begin{aligned}
\lambda(\mathbf{x}) & =\left[\lambda_{1}(\mathbf{x}), \ldots, \lambda_{N}(\mathbf{x})\right], \\
\gamma & =\left[\gamma\left(\mathbf{x}-\mathbf{x}_{1}\right), \ldots, \gamma\left(\mathbf{x}-\mathbf{x}_{N}\right)\right]^{T}, \\
f & =\left[f_{0}(\mathbf{x}), \ldots, f_{p}(\mathbf{x})\right]^{T}, \\
{[\boldsymbol{\Gamma}]_{i, j} } & =\gamma\left(\mathbf{x}_{i}-\mathbf{x}_{j}\right), \\
{[\mathbf{F}]_{l, j} } & =f_{l}\left(\mathbf{x}_{j}\right), \\
\mathbf{H} & =\left(\mathbf{F}^{T} \boldsymbol{\Gamma}^{-1} \mathbf{F}\right)^{-1}, \\
g & =\left(f-\mathbf{F}^{T} \boldsymbol{\Gamma}^{-1} \gamma\right) .
\end{aligned}
$$

The pixels at the CCD camera are regularly distributed, $\mathbf{x}_{i, j}=\mathbf{x}_{0,0}+(i \Delta x, j \Delta y)$. Then $\boldsymbol{\Gamma}$ is a symmetric Toeplitz matrix. Its analytical inversion is a subject of research, and it has been solved only for a number of canonical cases. ${ }^{19}$ Therefore Eq. (8) can be computed numerically, by use of several optimized techniques. ${ }^{20,21}$ As a consequence of being a symmetric matrix, the functions $\lambda_{1}(\mathbf{x}), \ldots, \lambda_{N}(\mathbf{x})$ are all the same except for a shift

$$
\lambda_{i}(\mathbf{x})=\Lambda(\mathbf{x}) * \delta\left(\mathbf{x}-\mathbf{x}_{i}\right),
$$

where $*$ means convolution, $\delta(x)$ is the Dirac-delta function, and kriging interpolation given by Eq. (5) is transformed in a convolution process ${ }^{17}$ :

$$
Z(\mathbf{x})=\Lambda(\mathbf{x}) * \Pi(\mathbf{x}),
$$

where $\Pi(\mathbf{x})=\sum_{i=1}^{N} \delta\left(\mathbf{x}-\mathbf{x}_{i}\right) Z_{i}$.

\section{Interpolation Uncertainty}

One calculates the uncertainty by introducing Eq. (5) into Eq. (6), which results in

$$
\sigma^{2}(\mathbf{x})=\gamma^{T} \Gamma^{-1} \gamma-g^{T} \mathbf{H} g .
$$

Under this approach, kriging is exact, which means that the estimation at locations $\mathbf{x}_{i}$ at which data are obtained is exactly the measured value $Z_{i}$. When noise is not negligible, an exact fit of the interpolated values to the experimental data is inconvenient.

It also happens that the uncertainty estimated by kriging is not coincident with the standard procedure of uncertainty estimation given by Eq. (1). This can be seen in the following way: When only one data value is obtained, it is easy to solve Eq. (11) because vectors and matrices become numbers, with the result that the estimated uncertainty for this simple case $^{22}$ is $\sigma^{2}(\mathbf{x})=2 \gamma(\mathbf{x})$. It is also known that the value of the variogram at the origin is equal to the variance of the random noise, ${ }^{23} \gamma(0)=s^{2}$. Therefore the uncertainty estimated by kriging at the location at which data are performed is $\sigma(\mathbf{0})=\sqrt{2} s$. However, with Eq. (1), this value should be $I^{2}+s^{2}$. As a consequence, the following modifications to kriging equations,

$$
\begin{gathered}
\gamma \rightarrow \bar{\gamma}=\gamma-\frac{\gamma(0)}{2}, \\
\Gamma_{i, j} \rightarrow \bar{\Gamma}_{i, j}=\Gamma_{i, j}-\gamma(0) \delta_{i, j}-I_{i} I_{j},
\end{gathered}
$$

have been performed to filter noise, to include the resolution of the measuring, and to make the uncertainty estimation by kriging coincident with the case of experimental data with no spatial dependence, ${ }^{13}$ where $\delta_{i, j}$ is the $i$ Kronecker and $I_{i}$ is the resolution of the $i$ th measuring device. Then the uncertainty, when only one data value has been obtained, is

$$
\sigma_{1}^{2}(\mathbf{x})=2 \bar{\gamma}-1 / \bar{\Gamma}_{i, j}^{-1}=2 \gamma(\mathbf{x})-\gamma(0)+I_{0}^{2},
$$

and the uncertainty at the location of the measuring device is $\sigma_{1}^{2}(\mathbf{0})=s^{2}+I_{0}^{2}$, equal to the uncertainty calculated with Eq. (1) for $N=1$.

Now let us see how kriging performs the interpolation and calculates the uncertainty in terms of the sampling frequency and the other metrological parameters. As an example, we have simulated a sinusoidal signal $[f(x)=\sin (2 \pi x)]$ with two random processes:

$$
Z_{i}=f\left(x_{i}\right)+p_{1}+p_{2},
$$

where $p_{1}$ is a zero-mean additive Gaussian probability distribution with standard deviation $s$, representing the random fluctuations of the measured quantity, and $p_{2}$ is an additive uniform probability distribution between $\left[-A_{i}, A_{i}\right]$, representing the resolution of the measuring devices whose standard deviation is $I_{i}=A_{i} / \sqrt{3}$.

In Figs. 1-3 the interpolation and uncertainty are calculated for three selected cases with different random fluctuations and sampling frequencies. When the sampling frequency $v$ is low, as well as the amplitude of the random fluctuations $(v=2.5, s$ $=0.001$, and $I=0.001$ ), the uncertainty at the sam- 


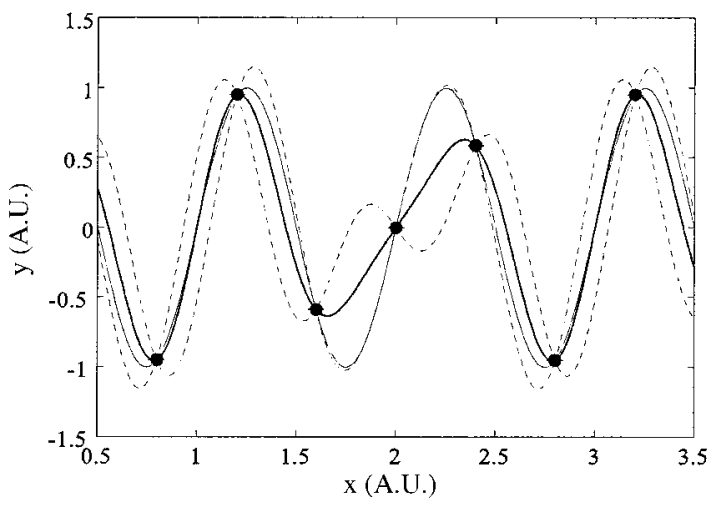

(a)

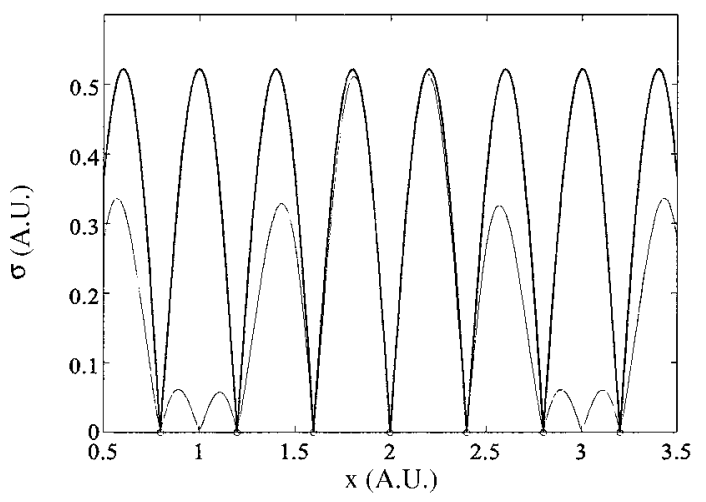

(b)

Fig. 1. (a) Estimation by kriging for a quantity with spatial dependence $f(x)=\sin (2 \pi x)$ when it is sampled with a frequency $v$ $=2.5$. Solid curve, kriging estimation; dashed curve, noiseless simulation; dotted curve, error bands; circles, simulated measurements; I, resolution of the measuring devices. Here $s=0.001, I_{0}$ $=0.001$. (b) Estimated (thick curve) and real (thin curve) uncertainties. We can see that there is a strong fluctuation in uncertainty estimation.

pling locations is approximately that of the case with nonspatial dependence [Eq. (1)], $\sigma \simeq\left(s^{2}+I^{2}\right)^{1 / 2}$ $=0.0014$, as it is shown in Fig. 1. Owing to the poor sampling, the spatial correlation cannot provide information at locations between the data values and the uncertainty at these locations increases considerably. On the other hand, when the sampling frequency increases (Fig. 2; $v=30$, and the rest of parameters are the same as those of Fig. 1), the spatial correlation is at work and makes the uncertainty decrease everywhere, not only where sampling is performed. In Fig. 3 the frequency is also $v=30$, but now the random fluctuations are greater $\left(s=0.3, I_{0}\right.$ $=0.1$ ). The spatial correlation is still able to decrease the uncertainty much more than the theoretical nonspatially correlated value $\left(s^{2}+I^{2}\right)^{1 / 2}$ at all locations, but it is always greater than $I$.

\section{Comparison with Nyquist Criterion}

Now that we know how the uncertainty calculated with kriging behaves when a regular sampling is performed in some limit cases, we will analyze the uncertainty in terms of the sampling frequency $v$, the

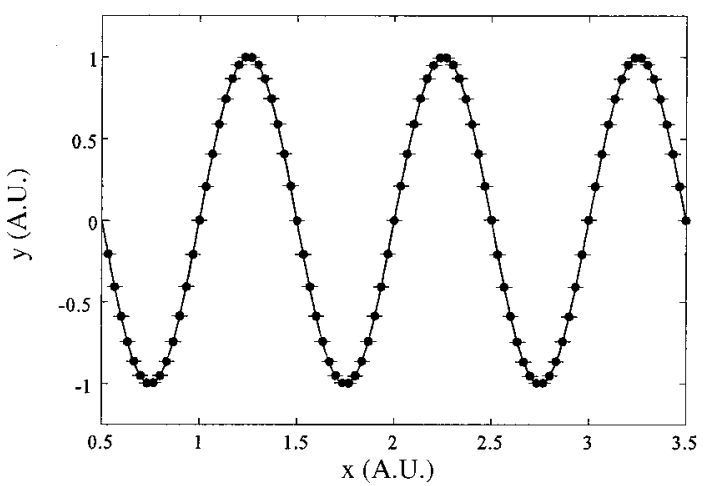

(a)

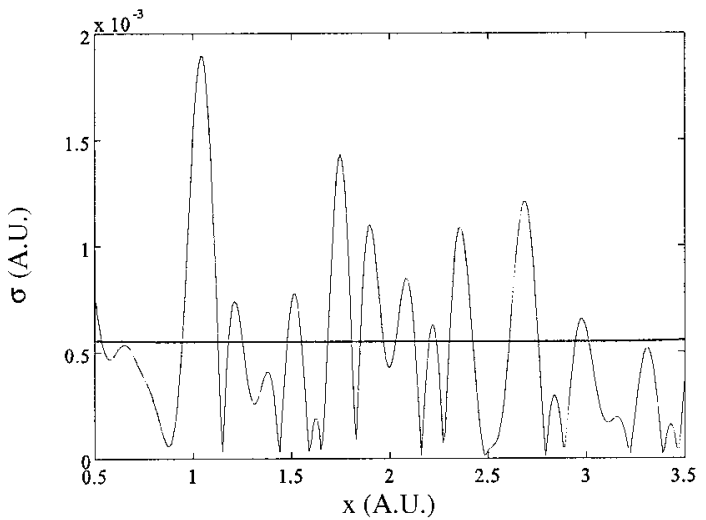

(b)

Fig. 2. (a) Estimation by kriging for a quantity with spatial dependence $f(x)=\sin (2 \pi x)$ when it is sampled with a frequency $v$ $=30$. Solid curve, kriging estimation; dashed curve, noiseless simulation; dotted curve, error bands; circles, simulated measurements; I, resolution of the measuring devices. The parameters for the measuring process are $s=0.001, I_{0}=0.001$. (b) Estimated (thick curve) and real (thin curve) uncertainties. Now the estimated uncertainty is much lower and does not present fluctuations.

random fluctuations $s$, and the resolution of the measuring devices $I$. For this, we have simulated the same process of Eq. (15), and we have defined the following parameter:

$$
\Sigma(\nu, \bar{s}, \bar{I})=\max [|\bar{\sigma}(\mathbf{x})|]_{v, s, I}
$$

which is closely related to the signal-to-noise ratio as a measurement of the interpolation quality. Here $v$ is the frequency in fringes per period, and the horizontal bar over the parameter means normalization with respect to the amplitude of the signal $A=\{\max [|f(\mathbf{x})|]-\min [|f(\mathbf{x})|]\} / 2$ (i.e., $\bar{s}=s / A$ ).

Among all the possible elections for $f(\mathbf{x})$, to calculate $\Sigma(v, \bar{s}, \bar{I}$ we have chosen the functions $f(x)$ $=\sin (2 \pi x)$ for one-dimensional (1D) images and $f(x, y)=\sin (2 \pi x) \sin (2 \pi y)$ for $2 \mathrm{D}$ images. This election is based on the fact that any band-limited function can be described as a combination of sine functions. For more general band-limited images with noise, we approximate that $\left.\max [\bar{\sigma}|(\mathbf{x})|]\right|_{v, s, I}$ is that of the sine 


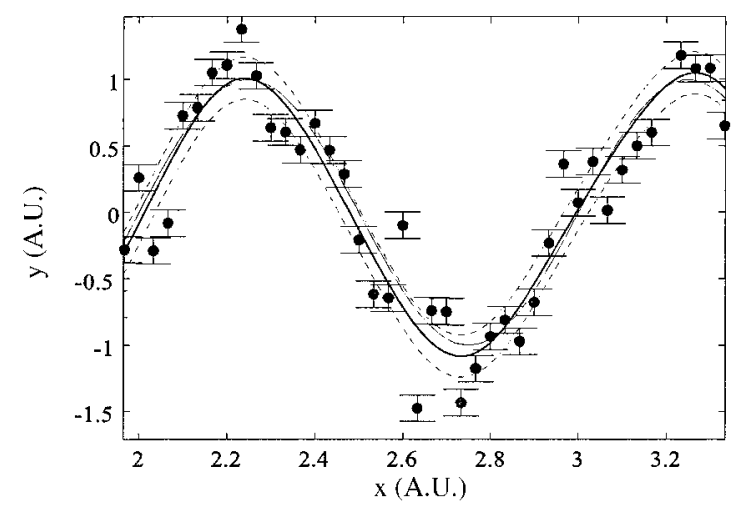

(a)

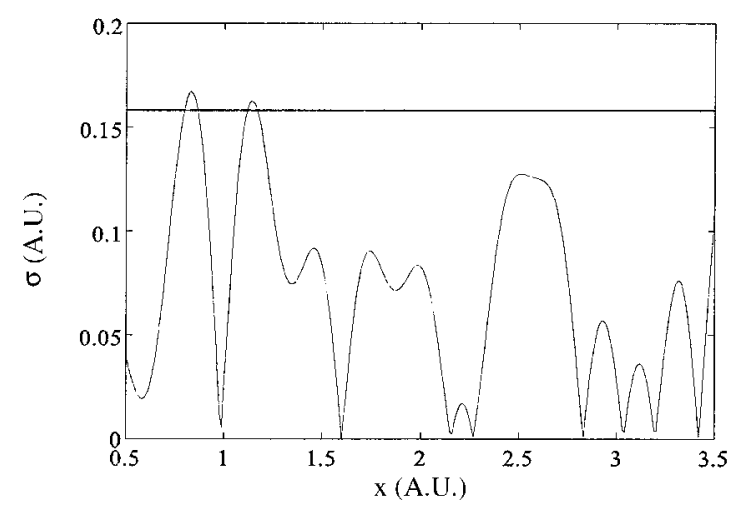

(b)

Fig. 3. (a) Estimation by kriging of a quantity with spatial dependence $f(x)=\sin (2 \pi x)$ when it is sampled with a frequency $v$ $=30$. Solid curve, kriging estimation; dashed curve, noiseless simulation; dotted curve, error bands; circles, simulated measurements; I, resolution of the measuring devices. Here $s=0.3, I_{0}$ $=0.1$. (b) Estimated (thick curve) and real (thin curve) uncertainties. Owing to the sampling, uncertainty decreases considerably (approximately 0.15), but it is higher than resolution $I_{0}$.

function whose frequency $v$ is the maximum frequency of the image, without considering noise.

In Fig. 4, $\Sigma(v, \bar{s}, \bar{I})$ is shown for $f(x)=\sin (2 \pi x)$ and $\bar{I}=0.001$. The parameter $\Sigma(\nu, \bar{s}, \bar{I})$ decreases when the sampling frequency increases and the random fluctuations decrease, coinciding with Figs. 1-3. For frequencies $v \leq 2$, the interpolation is not satisfactory for any value of $\bar{s}$, and $\Sigma(v \leq 2, \bar{s}) \geq 0.75$. However, when $\bar{s}$ is small, $\Sigma$ decreases considerably for frequencies $v \geq 5$ (Fig. 5). This decreasing of the uncertainty with the sampling frequency can be compared with the Nyquist criterion for the reconstruction of regularly sampled signals. According to such criterion, when the WKS method is used, the interpolation is exact for frequencies higher than $v \geq 2$, and the signal cannot be reconstructed at all for lower frequencies. When the kernel obtained with kriging is used, the transition between good to poor sampling is not abrupt but smooth. When $\bar{s}$ is high, $\Sigma$ also decreases when the sampling frequency increases but to a lower rate.

With kriging, 2D images are interpolated in the

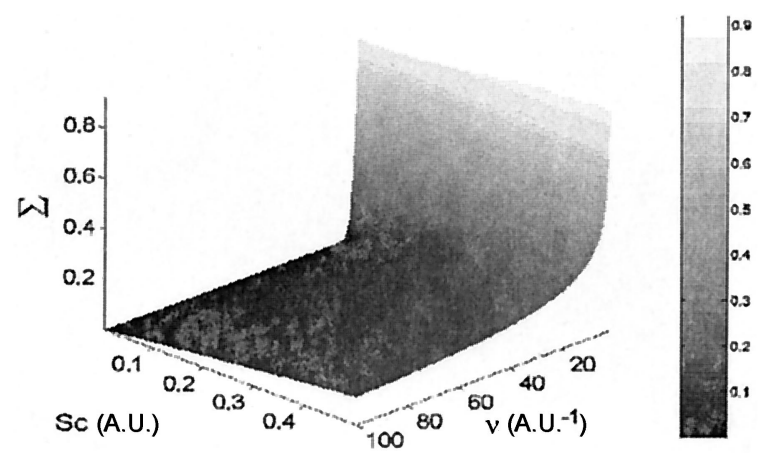

(a)

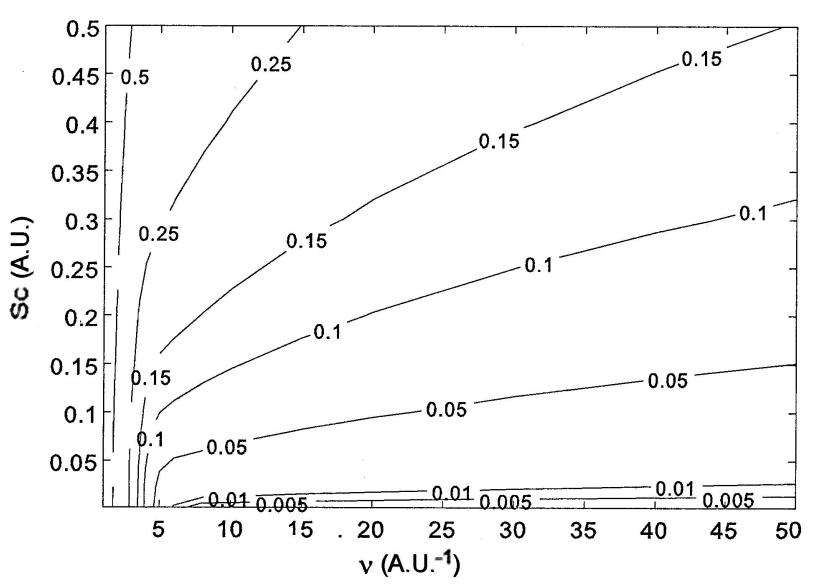

(b)

Fig. 4. (a) $\Sigma(v, \bar{s}, \bar{I})$ parameter for the $1 \mathrm{D}$ signal $f(x)=\sin (2 \pi x)$ when it is sampled with different frequencies and random fluctuations. (b) Contour.

same way as 1D signals, because equations for calculating $\lambda(\mathbf{x})$ and $\sigma(\mathbf{x})$ are independent of the dimension when the variogram is considered isotropic. We have performed the same process, but now $f(\mathbf{x})$ $=\sin (2 \pi x) \sin (2 \pi y)(\bar{I}=0.001)$, and the parameter $\Sigma(v, \bar{s}, \bar{I})$ for such a 2D image is shown in Fig. 6. It

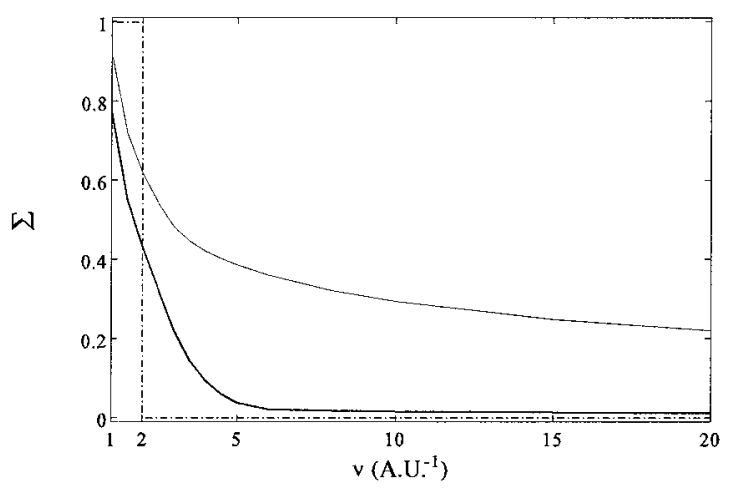

Fig. 5. Plot showing how $\Sigma$ decreases with the sampling frequency for two values of the random fluctuations: $\bar{s}=0.5$ (thin curve) and $\bar{s}=0$ (thick curve). When $\bar{s}$ is small, $\Sigma$ decreases strongly between $v=2$ and 5 . (arb. u. $)^{-1}$. When $\bar{s}=0.5, \Sigma$ decreases more gradually. Dashed lines represent the uncertainty for Nyquist criterion. 


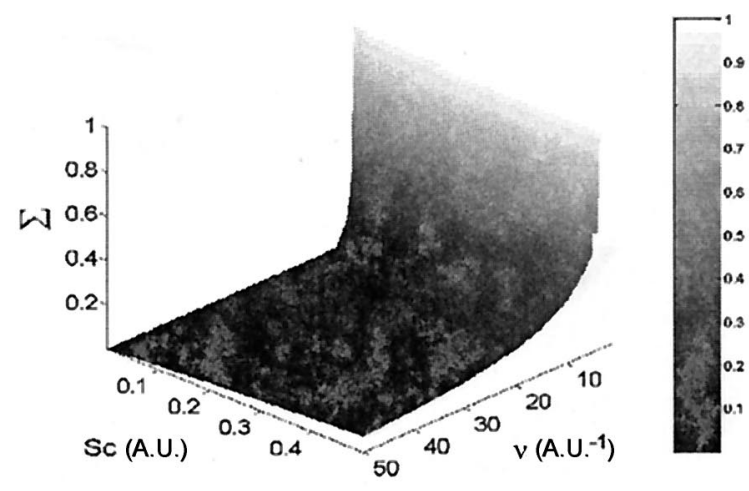

(a)

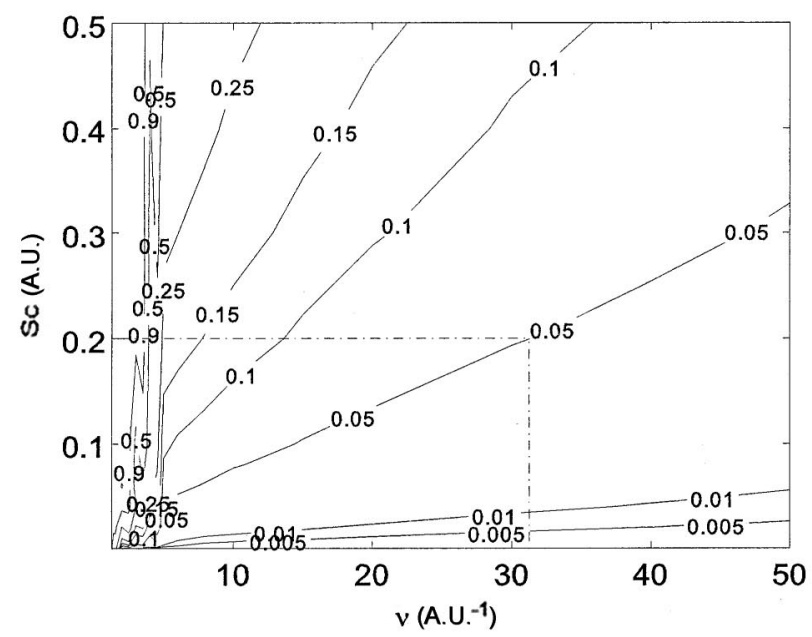

(b)

Fig. 6. (a) $\Sigma(v, \bar{s}, \bar{I})$ parameter for the 2D image $f(x)$ $=\sin (2 \pi x) \sin (2 \pi y)$ when it is sampled with different frequencies and random fluctuations. (b) Contour.

shows results quite similar to that of the $1 \mathrm{D}$ image. There are some differences, though: The estimated uncertainty is lower for the same values of spatial correlation and sampling in that there are more data around a given position $\mathbf{x}$ [Fig. 6(b)]. For small values of $\bar{s}$, the decreasing of $\Sigma$ at the interval $v=2-5$ is steeper than for the 1D signal (Fig. 7), which is much similar to Nyquist criterion.

In this description we have assumed that the resolution of the measuring devices is low (although different from 0 because it might cause problems of stability in the inversion of $\bar{\Gamma}$ ). When the resolution $I$ is not negligible, we have numerically found that the $\Sigma$ parameter can be calculated from that of $\Sigma$ at $I$ $=0$ by using

$$
\Sigma(\nu, \bar{s}, \bar{I})=\left[\sum(\nu, \bar{s}, \bar{I}=0)^{2}+\bar{I}^{2}\right]^{1 / 2} .
$$

As an example, in Fig. 8, $\Sigma(v, \bar{s}, \bar{I})$ is shown for $f(\mathbf{x})$ $=\sin (2 \pi x)$, for different values of $\bar{s}$ and $\bar{I}$ (with $v$ $=50$ ), by use of kriging equations [Eq. (16) and (17)]. As can be observed, the numerical results by use of both equations $\Sigma(\nu, \bar{s}, \bar{I})$ are similar.

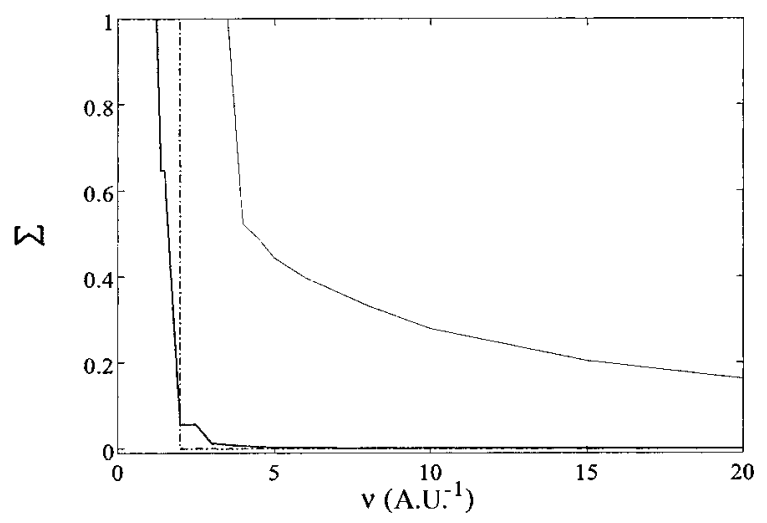

Fig. 7. Plot showing how $\Sigma$ decreases with sampling frequency for two values of the random fluctuations: $s=0.5$ (thin curve) and $s$ $=0$ (thick curve). When $s$ is small, $\Sigma$ decreases strongly between $v=2$ and 5 (arb. u. $)^{-1}$. When $s=0.5, \Sigma$ decreases more gradually. Dashed lines represent the uncertainty for the Nyquist criterion.

\section{Experimental Results}

We have applied kriging to experimental 2D images. In Fig. 9 we can see a typical image that belongs to the fringe pattern of a $350-\mu \mathrm{m}$ defect on a metallic plate [Fig. 9(a)] by use of the shadow moiré technique. ${ }^{24}$ We have calculated the variogram for this image by using Eq. (2), but we have used only the data at the diagonal $(N=512)$ because, when all the points are considered $(512 \times 512=262,144)$, its calculation is quite time consuming. The value of the variogram at the origin is $\gamma(0)=24.0$ gray-level (g.l.) units, and therefore the standard deviation is $s$ $=\sqrt{\gamma(0)}=4.9$ g.l. ${ }^{23}$ where the camera resolution $I$ $=1 \mathrm{~g} . \mathrm{l}$. We have calculated $\Lambda(x)$-Fig. 10 - and $\sigma(x)$ by means of Eqs. (10) and (11), respectively, where we have used a quadratic model for $f_{l}(\mathbf{x})(l=1, \ldots, 5)$ in Eq. (3): $x, y, x^{2}, y^{2}, x y$. The maximum uncertainty when the interpolation is performed with kriging is 3.23 g.l., and the uncertainty without considering spatial correlation is $\left(s^{2}+I^{2}\right)^{1 / 2}=5.0$ g.l. In that kriging considers the spatial correlation for perform-

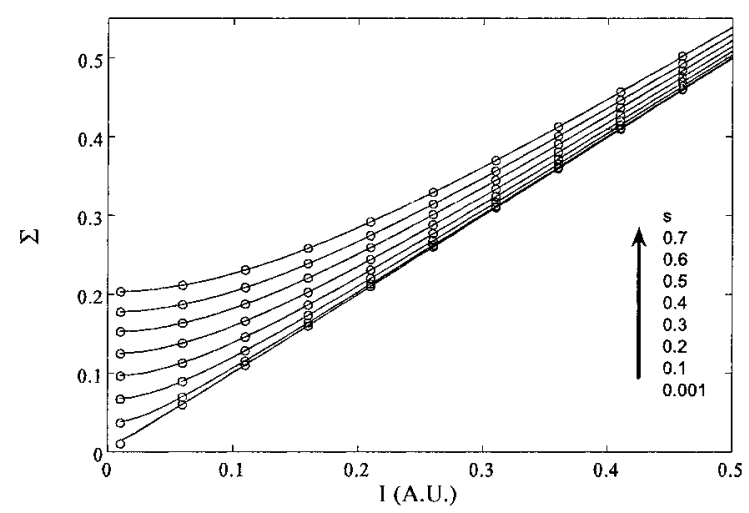

Fig. 8. $\Sigma(v, \bar{s}, \bar{I})$ parameter for several values of $\bar{s}$ and $\bar{I}$. Solid curves are estimated with Eq. (17), and dots are obtained with the general expression for kriging [Eq. (16)]. The function used to obtain this result is $f(\mathbf{x})=\sin (2 \pi x)$, with a sampling frequency $v$ $=50$. 


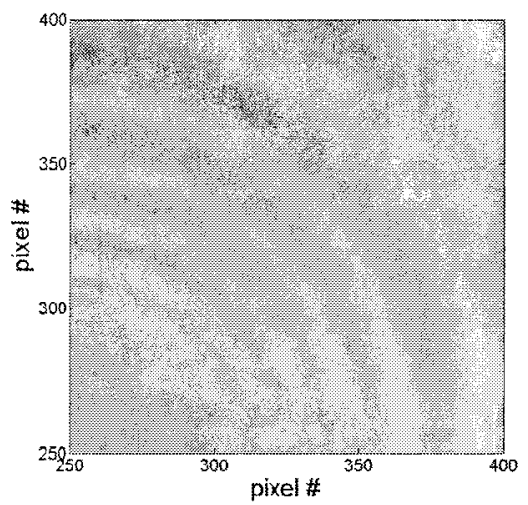

(a)

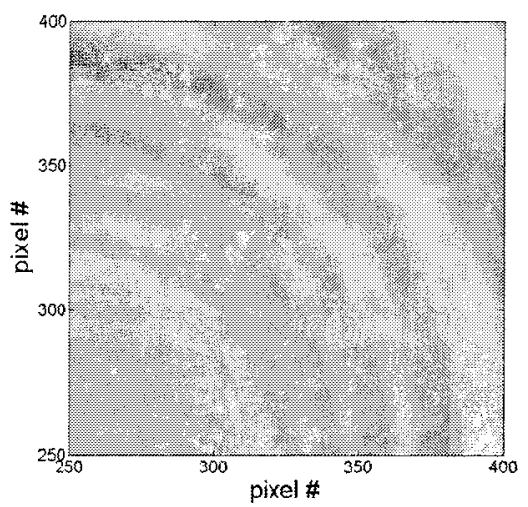

(b)

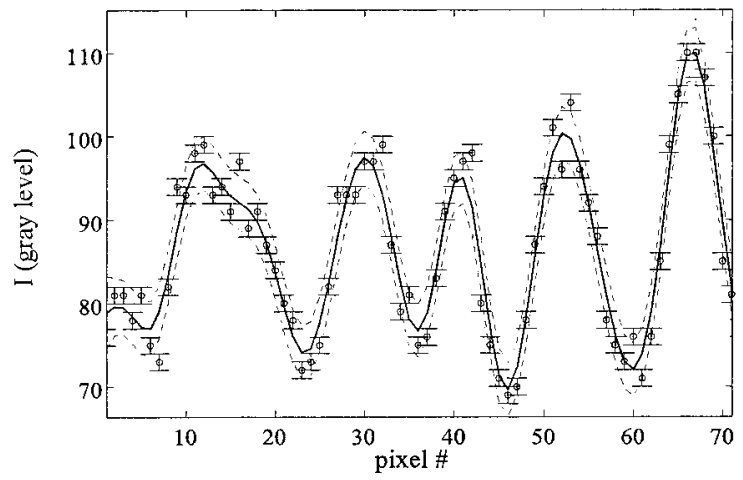

(c)

Fig. 9. (a) Two-dimensional image obtained with a CCD camera consisting of the fringe pattern of a $350-\mu \mathrm{m}$ defect obtained with the shadow moiré technique. It can be observed that the image presents noise. (b) Same 2D image of (a) after it was processed with kriging. (c) Profile of (a) and (b) showing the experimental data (circles), interpolation (solid curve), and the error bars (dashed curve).

ing the interpolation, it is able to decrease the uncertainty when the sampling frequency is high enough.

In Fig. 9(b) the interpolation performed by kriging is shown, and, in Fig. 9(c), a profile of such interpolation can be seen along with the experimental data. It is clear that the interpolation does not present short-range fluctuations, and the uncertainty bands covers the experimental data quite efficiently.

In some circumstances, there is a need to limit the

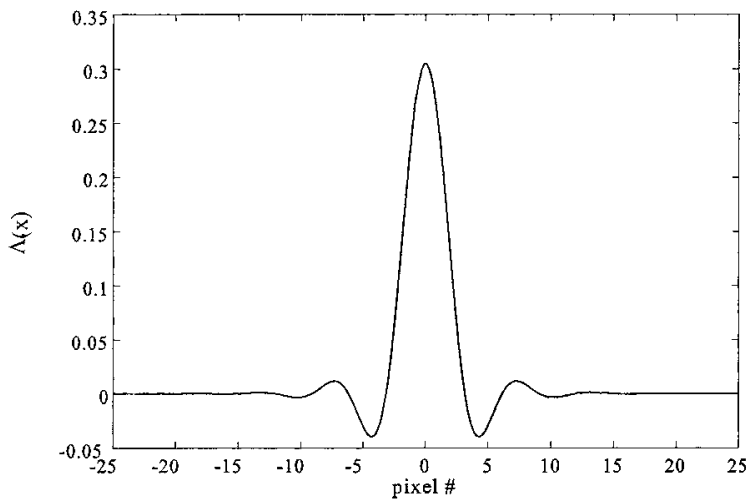

Fig. 10. Profile of the convolution kernel $\Lambda$ for the interpolation of Fig. 9(a) obtained with Eq. (8). Because we have considered the quantity to be isotropic, the convolution kernel presents revolution symmetry.

maximum uncertainty when the image is interpolated. There are several solutions to adopt, such as to improve the experimental conditions reducing the random fluctuations, to change the camera for another with better resolution or, normally the best option, to increase the sampling frequency by adjustment of the magnification. Because an increase in the magnification reduces the field of view, a compromise is required.

A procedure to determine the minimum sampling frequency so that the interpolation uncertainty estimated with kriging will be lower that a threshold value is to use the $\Sigma(v, \bar{s}, \bar{I})$ parameter. Let $\sigma_{\max }$ be the maximum uncertainty admitted for an image. Then $\Sigma(\nu, \bar{s}, \bar{I})=\sigma_{\max } / A$, and, by simply inverting this equation, $v=\Sigma^{-1}\left(\sigma_{\max } / A, \bar{s}, \bar{I}\right)$, one obtains the minimum sampling frequency. For example, let us have a $2 \mathrm{D}$ image whose amplitude $A=2$ and $s=0.4(\vec{s}$ $=0.2)$. If we want to have an uncertainty of $\sigma(\mathbf{x})$ $=0.1(\bar{\sigma}=0.05)$, and, by looking at Fig. $6(\mathrm{~b})$ (dasheddotted lines), we find that the sampling frequency required is $v=31$ samples per period.

Now let us see how accurate is the approximation of using $\Sigma(v, \bar{s}, \bar{I})$ calculated with the sine function for the uncertainty calculation of experimental fringes images. For this, we use the image of Fig. 9. The mean amplitude of the fringes of Fig. 9(a) is $A$ $=20 \mathrm{~g} . \mathrm{l}$. Thus the normalized noise is $\bar{s}=0.24$, and $\Sigma(\nu=12, \bar{s}=0.24, \bar{I}=0) \approx 0.15$. Therefore the maximum estimated uncertainty, computed with Eq. (17), is $\sigma=A \Sigma(v=12, \bar{s}=0.24$, and $\bar{I}=0.05)$ $=3.16$ g.l., which is in good agreement with the uncertainty $\sigma=3.23$ g.l. calculated with Eq. (11).

\section{Conclusions}

In this paper we have analyzed the properties and advantages that kriging presents for image processing and noise filtering. When the experimental data are regularly sampled, kriging interpolation is solved as a convolution, and the convolution kernel depends on the metrological parameters, such as the random fluctuations of the measured quantity, the resolution 
of the measuring devices, the sampling frequency, and the spatial correlation. Kriging provides a method for calculating the uncertainty at interpolation, which is important when images are used for optical metrology because it allows us to determine the error in the estimation of the parameters measured with the image. We have also seen that the uncertainty proposed by kriging is lower when the sampling frequency is increased and when the random fluctuations are decreased. The results have been compared with Nyquist criterion, and a technique for determining the sampling frequency required to make the interpolation uncertainty lower than a threshold value has been proposed.

The authors thank Agustín González-Cano, Juan Antonio Quiroga, and Jesus Zoido for their help and fruitful discussions.

\section{References}

1. W. K. Pratt, Digital Image Processing (Wiley, New York, 1978).

2. H. P. Urbach, "Generalised sampling theorem for band-limited functions," Math. Comput. Modell. 38, 133-140 (2003).

3. A. Stern and B. Javidi, "Sampling in the light of Wigner distribution,” J. Opt. Soc. Am. A 21, 360-366 (2004).

4. A. Stern and B. Javidi, "Analysis of practical sampling and reconstruction from Fresnel fields," Opt. Eng. 43, 239-250 (2004).

5. C. E. Shannon, "Communication in presence of noise," Proc. IRE 37, 20-21 (1949).

6. G. C. Holst, CCD Arrays, Cameras, and Displays (SPIE, Bellingham, Wash., 1996).

7. A. J. Jerri, "The Shannon sampling theorem-its various extensions and applications," Proc. IEEE 65, 1565-1596 (1977).

8. K. F. Cheung and R. J. Marks II, "Imaging sampling below the Nyquist density without aliasing," J. Opt. Soc. Am. A 7, 92-105 (1990).

9. M. Pawlak and U. Stadmüller, "Recovering band-limited sig- nals under noise," IEEE Trans. Inf. Theory 42, 1425-1438 (1996).

10. M. Unser, "Sampling—50 years after Shannon," Proc. IEEE 88, 569-587 (2000).

11. P. Bevington, Data Reduction and Error Analysis for the Physical Sciences (McGraw-Hill, New York, 1969).

12. ISO, Guide to the Expression of the Uncertainty in Measurement (International Organization for Standardization, Geneva, Switzerland, 1995).

13. R. Christiensen, Linear Models for Multivariate, Time Series, and Spatial Data (Springer-Verlag, Berlin, 1985).

14. N. Cressie, Statistics for Spatial Data (Wiley, New York, 1991).

15. E. Bernabeu, I. Serroukh, and L. M. Sanchez-Brea, "A geometrical model for wire optical diffraction selected by experimental statistical analysis," Opt. Eng. 38, 1319-1325 (1999).

16. D. Mainy, J. P. Nectoux, and D. Renard, "New developments in data processing of noisy images," Mater. Charact. 36, 327-334 (1996).

17. W. Y. V. Leung, P. J. Bones, and R. G. Lane, "Statistical interpolation of sampled images," Opt. Eng. 40, 547-553 (2001).

18. T. D. Pham and M. Wagner, "Image enhancement by kriging and fuzzy sets," Int. J. Pattern Recognit. 14, 1025-1038 (2000).

19. G. Y. Hu and R. F. O'Connell, "Analytical inversion of symmetric tridiagonal matrices," J. Phys. A 29, 1511-1513 (1996).

20. G. S. Ammar and W. B. Gragg, "Superfast solution of real positive definite Toeplitz systems," SIAM J. Matrix Anal. Appl. 9, 61-76 (1988).

21. W. H. Press, S. A. Teukolski, W. T. Vetterling, and B. P. Flannery, Numerical Recipes in C (Cambridge U. Press, New York, 1992).

22. J. P. Chilès and P. Delfiner, Geostatistics (Wiley, New York, 1999).

23. L. M. Sanchez-Brea and E. Bernabeu, "On the standard deviation in charge-coupled device cameras: a variogram-based technique for nonuniform images," J. Electron. Imaging 11, 121-126 (2002).

24. G. Cloud, Optical Methods of Engineering Analysis (Cambridge U. Press, Cambridge, UK, 1998). 